\title{
Rational solitons in deep nonlinear optical Bragg grating
}

\author{
H. Alatas, ${ }^{1,2, *}$ A. A. Iskandar, ${ }^{1}$ M. O. Tjia, ${ }^{1}$ and T. P. Valkering ${ }^{3}$ \\ ${ }^{1}$ Department of Physics, Institut Teknologi Bandung, Jl. Ganesha 10, Bandung 40132, Indonesia \\ ${ }^{2}$ Theoretical Physics Laboratory, Department of Physics, Institut Pertanian Bogor, Jl. Meranti, Kampus IPB Darmaga, Bogor 16680, \\ Indonesia \\ ${ }^{3}$ Faculty of Applied Physics, University of Twente, P.O. Box 217, 7500 AE Enschede, The Netherlands
}

(Received 11 October 2005; published 6 June 2006)

\begin{abstract}
We have examined the rational solitons in the Generalized Coupled Mode model for a deep nonlinear Bragg grating. These solitons are the degenerate forms of the ordinary solitons and appear at the transition lines in the parameter plane. A simple formulation is presented for the investigation of the bifurcations induced by detuning the carrier wave frequency. The analysis yields among others the appearance of in-gap dark and antidark rational solitons unknown in the nonlinear shallow grating. The exact expressions for the corresponding rational solitons are also derived in the process, which are characterized by rational algebraic functions. It is further demonstrated that certain effects in the soliton energy variations are to be expected when the frequency is varied across the values where the rational solitons appear.
\end{abstract}

DOI: 10.1103/PhysRevE.73.066606

PACS number(s): 42.65.Tg

\section{INTRODUCTION}

The localization of light in the nonlinear Bragg grating has attracted the attention of a number of researchers since the discovery by Chen and Mills in their numerical work [1] of the so-called gap soliton solutions in the form of bright solitons existing within the linear band gap. This new found optical phenomenon was not only challenging theoretically, it was also observed experimentally [2], leading to further study on its potential novel function for optical gate applications [3]. The work of Chen and Mills was followed by an analytic study of the stationary phenomena with zero energy flow by Trulinger and Mills [4]. Based on the coupled mode theory they have derived the coupled ordinary differential equations of the slowly varying forward and backward electric field envelopes. This formulation was applied to the case of shallow nonlinear Bragg grating, for which the linear as well as the nonlinear refractive index modulations in the grating were assumed to be small compared to their respective averaged value. This condition allows one to assume that a superposition of two simple forward and backward plane waves is sufficient to describe the field dynamics. In the following investigations, the moving gap soliton, which is a solution to the time dependent coupled equations, was first obtained in a limiting case by Christodoulides and Joseph [5], while the full solutions were found later by Aceves and Wabnitz [6]. Subsequently Feng and Kneubühl [7] have found that, in addition to the gap solitons, a bounded solution could exist outside the linear band gap in the form of out-gap dark and antidark soliton solutions. Conti and Trillo [8] have explained the existence of all these in-gap and out-gap solitons on the basis of a simple geometrical analysis. They found in their work that the in-gap and out-gap solitons are degenerated at the band edges into a special class of rational

*Electronic address: alatas@ipb.ac.id solitonic profiles, namely, the Lorentzian soliton, with the associated form expressed by an algebraic Lorentzian function. They have predicted that this special solitonic solution would play a significant role in the localization of light in periodic media since its appearance was also found in a periodic system with photonic band gap of nonlinear origin [9]. The appearance of rational solitons was also reported in another optical system, e.g., optical fiber with gain and spectral filtering governed by the generalized Ginzburg-Landau equation [10].

In order to deal with a more general model than the shallow grating models described above, with the nonlinear modulation allowed to be "deep," i.e., of the same order as its average, the plane wave assumption has to be relaxed. Meanwhile, at least two different methods have been proposed for the derivation of the corresponding governing equations. The first method was formulated by Sipe et al. [11]. In their formulation, the general Bloch functions was used as the fundamental waves. The coupled differential equations were derived from the superposition of two dominant Bloch waves, while the other Bloch functions were treated perturbatively. The second method was introduced by lizuka and de Sterke [12], with its modification given by Alatas et al. [13] for monochromatic waves. This method is based on asymptotic formalism where the electric field is expanded in terms of all plane wave harmonics, and on the assumption that there exist two harmonics associated with dominant forward and backward fields with order of unity, while the other harmonics are assumed to be small and contribute only small corrections to the corresponding coupled mode equations. Although the two methods differ in their detailed formulations, they lead to the same so-called generalized coupled mode equations, but indeed with different expressions for the coefficients.

The coupled equations of deep nonlinear Bragg grating have been shown to admit the conventional in-gap and outgap solitons similar to those found in shallow gratings. 
Additional solution in the form of double hump gap soliton, which is absent in the shallow case, was also found along with some new out-gap solutions $[11,12]$. Recently, we found that the coexistence of in-gap dark and antidark soliton solutions are also admitted in the corresponding coupled equations [13]. These dark and antidark solitons are associated with saddles in the phase portrait, and do not occur in the shallow case. We have further found the existence of rational solitons on the boundaries separating two regions of different soliton solutions in the parameter space. These solitons are called rational solitons because of their algebraic functional expressions, and they are expected to play an important role in the dynamical process of related solitonic transition. However, we stopped short of discussing the connection and transition among those solutions. In this paper, we describe in detail the detuning process where these stationary dark and antidark solitons evolve into dark and antidark rational solitons due to frequency detuning. The induced field dynamics is an important issue for the observation of solitons in finite grating systems since their existence also depends on this parameter.

\section{THE COUPLED MODE EQUATION AND THEIR FIXED POINTS}

As mentioned above, the basic formulation for the dynamics of the forward and backward envelope fields in deep nonlinear Bragg grating has been proposed previously in Refs. [11-13]. Our ensuing analysis in this work is carried out on the basis of the specific model proposed in [13], which, when applied to monochromatic waves, yields the following coupled equations:

$$
\begin{aligned}
& i \frac{d E_{F}}{d z}+\delta_{1} E_{F}+\delta_{2}\left[\left|E_{F}\right|^{2}+2\left|E_{B}\right|^{2}\right] E_{F}+\delta_{3}^{*} E_{B} \\
& \quad+\delta_{4}^{*}\left[2\left|E_{F}\right|^{2}+\left|E_{B}\right|^{2}\right] E_{B}+\delta_{4} E_{F}^{2} E_{B}^{*}+\delta_{5} E_{F}^{*} E_{B}^{2}=0, \\
& -i \frac{d E_{B}}{d z}+\delta_{1} E_{B}+\delta_{2}\left[\left|E_{B}\right|^{2}+2\left|E_{F}\right|^{2}\right] E_{B}+\delta_{3} E_{F} \\
& \quad+\delta_{4}\left[2\left|E_{B}\right|^{2}+\left|E_{F}\right|^{2}\right] E_{F}+\delta_{4}^{*} E_{B}^{2} E_{F}^{*}+\delta_{5}^{*} E_{B}^{*} E_{F}^{2}=0,
\end{aligned}
$$

where $E_{F}$ and $E_{B}$ are forward and backward envelopes of the dominant plane waves. The small coefficient $\delta_{1}$ is given by $\delta_{1}=\bar{\varepsilon}_{r}\left(\omega^{2} \alpha+\Delta\right) / 2 c^{2} N k_{B}$, where $\alpha=\omega^{2} \bar{\varepsilon}_{r} c^{-2} k_{B}^{-2} \sum_{j \neq-N, 0}\left|e_{j}\right|^{2} /$ $\left((2 j+N)^{2}-N^{2}\right)$ denotes the gap center correction, $\Delta=\omega^{2}$ $-N^{2} \omega_{B}^{2}$ the detuning frequency with $\omega_{B}=c k_{B} / \sqrt{\bar{\varepsilon}_{r}}$, and $k_{B}$ $=\pi / \Lambda$ denoting Bragg frequency and wave number, respectively, while $\Lambda$ is the periodicity of the grating, $\bar{\varepsilon}_{r}$ is the averaged dielectric constant, $N$ is the order of the stop band, and $e_{j}=\widetilde{\chi}_{j}^{(1)} / \bar{\varepsilon}_{r}$ with $\widetilde{\chi}_{j}^{(1)}$ representing the $j$ th Fourier coefficient of the linear susceptibility function. The parameter $\delta_{1}$ is the only operational parameter to be controlled externally. The coefficients $\delta_{i}$ 's with $i=2,3,4,5$ are small as well, and they are determined by the properties of the grating parameters consisting of the material and geometrical specifications of a deep nonlinear grating. The coefficient of the linear modulation term is given by $\delta_{3}=\omega^{2} \bar{\varepsilon}_{r}\left(e_{N}+\beta\right) / 2 c^{2} N k_{B}$, with $\beta=\omega^{2} \bar{\varepsilon}_{r} c^{-2} k_{B}^{-2} \quad \sum_{j \neq-N, 0} e_{-j} e_{j+N} /\left((2 j+N)^{2}-N^{2}\right)$ denoting the gap width correction. The coefficient of the self-phase and cross-phase modulation terms encompassed in $\delta_{2}$ is given by $\delta_{2}=3 \omega^{2} \bar{\chi}^{(3)} / 2 c^{2} N k_{B}$, with $\bar{\chi}^{(3)}$ representing the averaged third order susceptibility. The coefficients of the two nonlinear modulation terms in the equations are represented by $\delta_{4}$ and $\delta_{5}$, with $\delta_{4}=3 \omega^{2} \widetilde{\chi}_{N}^{(3)} / 2 c^{2} N k_{B}$ and $\delta_{5}=3 \omega^{2}\left(\tilde{\chi}_{2 N}^{(3)}+\gamma\right) / 2 c^{2} N k_{B}$, where $\tilde{\chi}_{N(2 N)}^{(3)}$ are the $N$ th (2Nth) Fourier coefficients of third order susceptibility function and $\gamma=\omega^{2} \bar{\chi}^{(3)} \tilde{\chi}_{2 N}^{(1)} / 8 c^{2} N^{2} k_{B}^{2} \bar{\varepsilon}_{r}$. Generally, the coefficients $\delta_{3}, \delta_{4}$, and $\delta_{5}$ are complex quantities, but in this study they are restricted to be real. Clearly, Eqs. (1) and (2) will reduce to those for the shallow nonlinear Bragg grating when $\alpha, \beta, \gamma, \delta_{4}$, and $\delta_{5}$ are set equal to zero [8]. The mathematical details of this formulation can be found in Refs. [12,13].

We shall hereforth focus our discussion on the case of zero energy flow, which implies that the fields $E_{F}$ and $E_{B}$ can be written as follows:

$$
E_{F}(z)=f(z) \exp (i \phi(z)), \quad E_{B}(z)=f(z) \exp (-i \phi(z)),
$$

where the amplitude $f(z)$ and phase $\phi(z)$ are real functions of z. Substituting Eq. (3) into Eqs. (1) and (2) yields the following coupled equations:

$$
\begin{aligned}
\frac{d \phi}{d z}= & \delta_{1}+3 \delta_{2} f^{2}+\delta_{3} \cos (2 \phi)+4 \delta_{4} f^{2} \cos (2 \phi) \\
& +\delta_{5} f^{2} \cos (4 \phi) \\
\frac{d f}{d z}= & \delta_{3} f \sin (2 \phi)+2 \delta_{4} f^{3} \sin (2 \phi)+\delta_{5} f^{3} \sin (4 \phi) .
\end{aligned}
$$

The corresponding Hamiltonian for these equations is given by

$$
\begin{aligned}
H\left(f^{2}, \phi\right)= & \delta_{1} f^{2}+\frac{3}{2} \delta_{2} f^{4}+\delta_{3} f^{2} \cos (2 \phi)+2 \delta_{4} f^{4} \cos (2 \phi) \\
& +\frac{1}{2} \delta_{5} f^{4} \cos (4 \phi),
\end{aligned}
$$

where $\left(f^{2}, \phi\right)$ constitutes the canonical pair in the phase plane, i.e., $d \phi / d z=\partial H / \partial f^{2}, d f^{2} / d z=-\partial H / \partial \phi$. It is readily proven that Hamiltonian (6) is a conserved quantity satisfying $d H / d z=0$, and hence $H\left(f^{2}, \phi\right)$ will be represented as a real constant $h$.

Using this property and $H=h$ for $f^{2}$ one finds

$$
f_{ \pm}^{2}(\phi)=\frac{-b(\phi) \pm \sqrt{b(\phi)^{2}+4 a(\phi) h}}{2 a(\phi)} .
$$

With this, we arrive at the following equation for $\phi$ :

$$
\frac{d \phi}{d z}= \pm \sqrt{b(\phi)^{2}+4 a(\phi) h},
$$

where $\quad a(\phi)=3 \delta_{2} / 2+2 \delta_{4} \cos (2 \phi)+\delta_{5} \cos (4 \phi) / 2 \quad$ and $b(\phi)=\delta_{1}+\delta_{3} \cos (2 \phi)$. Thus, solving this equation for given $h$ yields the complete solution.

To analyze the bifurcation process, it is convenient to express the Hamiltonian of Eq. (6) in a Cartesian coordinate by 
the transformation defined by $f^{2}=x^{2}+y^{2}, \cos ^{2}(\phi)=x^{2} /\left(x^{2}\right.$ $\left.+y^{2}\right), \sin ^{2}(\phi)=y^{2} /\left(x^{2}+y^{2}\right)$. As a result, the Hamiltonian can be recast into the following form:

$$
H(x, y)=\delta_{+} x^{2}+\delta_{-} y^{2}+A x^{4}+B y^{4}+2 C x^{2} y^{2}
$$

with the coefficients in Eq. (9) given in terms of those appearing in Eqs. (1) and (2) according to the following expressions:

$$
\begin{gathered}
\delta_{ \pm}=\delta_{1} \pm \delta_{3}, \\
A=\left(3 \delta_{2}+4 \delta_{4}+\delta_{5}\right) / 2, \\
B=\left(3 \delta_{2}-4 \delta_{4}+\delta_{5}\right) / 2, \\
C=3\left(\delta_{2}-\delta_{5}\right) / 2 .
\end{gathered}
$$

It is clear that $A, B$, and $C$ are parameters fixed by the chosen grating parameters, while $\delta_{ \pm}$may have a positive, negative, or zero value depending on the operational condition specified by $\delta_{1}$.

In order to identify a solitonic solution of the Hamiltonian (9), we first determine all of the fixed points $(\bar{x}, \bar{y})$ from the equations $\partial H / \partial x=0$ and $\partial H / \partial y=0$. There are three types of fixed points for this Hamiltonian, classified, respectively, by (1) $\left(\bar{x}_{1}=0, \bar{y}_{1}=0\right),(2)\left(\bar{x}_{2} \neq 0, \bar{y}_{2}=0\right)$ or $\left(\bar{x}_{2}=0, \bar{y}_{2} \neq 0\right)$, and (3) $\left(\bar{x}_{3} \neq 0, \bar{y}_{3} \neq 0\right)$, according to

$$
\begin{gathered}
\bar{x}_{2}^{2}=\bar{\xi}_{2} \equiv-\frac{\delta_{+}}{2 A}, \quad \bar{y}_{2}^{2}=\bar{\eta}_{2} \equiv-\frac{\delta_{-}}{2 B}, \\
\bar{x}_{3}^{2}=\bar{\xi}_{3} \equiv \frac{C \delta_{-}-B \delta_{+}}{2\left(A B-C^{2}\right)}, \quad \bar{y}_{3}^{2}=\bar{\eta}_{3} \equiv \frac{C \delta_{+}-A \delta_{-}}{2\left(A B-C^{2}\right)} .
\end{gathered}
$$

Clearly the fixed points exist in terms of newly introduced coordinates $(\xi, \eta)$ only if $\bar{\xi}_{n} \geqslant 0, \bar{\eta}_{n} \geqslant 0$. Since $\delta_{ \pm}$are linear in $\delta_{1}$, these conditions can, for fixed grating parameters, be met by varying the frequency through $\delta_{1}$. These fixed points are distinguished by their corresponding $h$ values of the Hamiltonian given below,

$$
\begin{gathered}
h_{1}=0, \\
h_{2 x}=\frac{1}{2} \delta_{+} \bar{\xi}_{2}, h_{2 y}=\frac{1}{2} \delta_{-} \bar{\eta}_{2}, \\
h_{3}=\frac{1}{2}\left(\delta_{+} \bar{\xi}_{3}+\delta_{-} \bar{\eta}_{3}\right) .
\end{gathered}
$$

It should be noted that for the shallow model where $\delta_{4}=\delta_{5}=0$, one finds the simple equality $A=B=C$, and hence no third type fixed points could exist as implied by Eq. (15).

\section{CLASSIFICATION OF BIFURCATION EVENTS}

Soliton solutions of Eqs. (1) and (2) are represented in the phase portraits of the Hamiltonian by the connections of a given saddle with itself or with another saddle.
The saddles are fixed points having certain stability characteristic determined in terms of its stability function. This function is found by evaluating the determinant of the Hessian of Hamiltonian (9), which leads to the following formulas:

$$
S=\frac{1}{4}\left[\frac{\partial^{2} H}{\partial x^{2}} \frac{\partial^{2} H}{\partial y^{2}}-\left(\frac{\partial^{2} H}{\partial x \partial y}\right)^{2}\right] .
$$

Specifically, $S<0$ corresponds to a saddle and $S>0$ to a center, while $S=0$ is associated with the bifurcation point. For the ensuing analysis of the bifurcation behaviors, the expressions of stability function for each type of fixed point must be determined. This is achieved by substituting Eqs. (14) and (15) into Eq. (19), with the result given as follows:

$$
\begin{gathered}
S_{1}=4 \delta_{+} \delta_{-}=4 A B \bar{\xi}_{2} \bar{\eta}_{2}, \\
S_{2 x}=\frac{8 \delta_{+}\left(C \delta_{+}-A \delta_{-}\right)}{A}=-4\left(A B-C^{2}\right) \bar{\xi}_{2} \bar{\eta}_{3}, \\
S_{2 y}=\frac{8 \delta_{-}\left(C \delta_{-}-B \delta_{+}\right)}{B}=-4\left(A B-C^{2}\right) \bar{\eta}_{2} \bar{\xi}_{3}, \\
S_{3}=\frac{16\left(C \delta_{-}-B \delta_{+}\right)\left(C \delta_{+}-A \delta_{-}\right)}{A B-C^{2}}=16\left(A B-C^{2}\right) \bar{\xi}_{3} \bar{\eta}_{3} .
\end{gathered}
$$

Clearly, in all cases, the bifurcation processes can be induced by tuning the frequency $\omega$ imbedded in $\delta_{1}$. From Eq. (23) one sees that the fixed points of the third type $\left(\bar{\xi}_{3}>0, \bar{\eta}_{3}\right.$ $>0$ ) are saddles if and only if $A B-C^{2}<0$, which is the case of our focus. It is also clear that the origin is a saddle if and only if $\delta_{+} \delta_{-}<0$, or equivalently $\left|\delta_{1}\right|<\left|\delta_{3}\right|$, which corresponds to the in-gap solution.

There are two distinct classes of bifurcation events, one involves only fixed points of the first and second type, while the second class involves the second and third type fixed points. The first class bifurcation occurs when

$$
S_{1}=S_{2 x}=0 \text { or } S_{1}=S_{2 y}=0 .
$$

Here, the change of stability of the origin coincides with the appearance of a new fixed point on the $x$ or $y$ axis. This bifurcation process can be best illustrated by the phase portraits for specific grating parameters, as will be presented below.

One possible bifurcation for the second case of Eq. (24) is demonstrated in Fig. 1. For this case, we have chosen $A<0, B<0$, a large positive $\delta_{+}$and small positive $C$ so that $\bar{\xi}_{2}>0, \bar{\xi}_{3}<0$, and $\bar{\eta}_{3}<0$ (absence of third type fixed point) while varying $\delta_{-}$from negative to positive. Then $S_{1}$ and $\bar{\eta}_{2}$ also change sign from negative to positive signifying the appearance of a saddle on the $y$ axis as $S_{2 y}$ changes from positive to negative. With $\bar{\xi}_{2}>0$ and $S_{2 x}>0$ the second type fixed point on the $x$ axis remains as a center throughout the process. Thus as a result of varying $\omega$ or $\delta_{-}$, the origin turns 


\section{$\stackrel{\text { Increasing } \omega}{\longrightarrow}$}
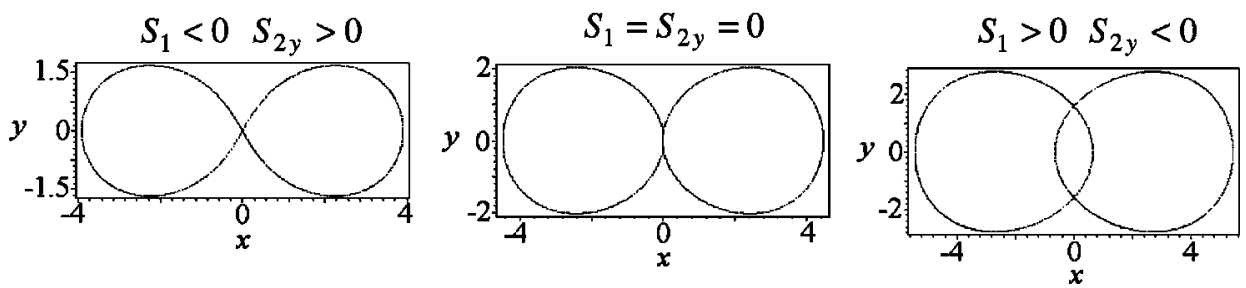

FIG. 1. A possible first class bifurcation process induced by increasing $\delta_{1}$, showing the level sets $h=h_{1} \quad$ (left graph), $h=h_{1}=h_{2 y}$ (middle graph), and $h=h_{2 y}$ (right graph). from a saddle into a center, and at the same time two saddles appear on the $y$ axis. One observes further in Fig. 1, the left graph is the well-known eight shape orbit, which represents a bright gap soliton [4]. This homoclinic connection evolves into a heteroclinic connection (right graph), which represents an out-gap dark or antidark soliton. In between, the process features the occurance of an orbit having infinite slopes at the origin (mid graph) which represents the rational soliton [8].

The second class bifurcation events occur when

$$
S_{2 x}=S_{3}=0 \text { or } S_{2 y}=S_{3}=0 .
$$

Consider for instance the second case with positive $\bar{\eta}_{2}$ representing the presence of a second type fixed point. A bifurcation occurs when the sign of $\bar{\xi}_{3}$ changes from negative to positive. The corresponding sign changes of $S_{2 y}$ and $S_{3}$ indicate that the second type fixed points turns from a saddle into a center, in concurrence with the appearance of a fixed points of the third type as a saddle.

Since the first class bifurcation events at the origin have been studied previously for the case of shallow grating model $[8,14]$, we shall henceforth concentrate our discussions on the second class process which involves the third type fixed point. As mentioned earlier, this fixed point is excluded in the case of shallow grating. For the following analysis, the Hamiltonian of Eq. (9) is rewritten in the $\xi$ - $\eta$ plane as $H(\xi, \eta)=\delta_{+} \xi+\delta_{-} \eta+A \xi^{2}+B \eta^{2}+2 C \xi \eta$, where $\xi=x^{2}$ and $\eta=y^{2}$, and $(\xi, \eta) \geqslant 0$. The heteroclinic connections are solutions of $H(\xi, \eta)-h_{3}=0$, which can be put into the factorized form as follows:

$$
\left(P_{1} \xi+Q_{1} \eta-h_{3}\right)\left(P_{2} \xi+Q_{2} \eta-h_{3}\right)=0,
$$

with

$$
\begin{array}{ll}
2 P_{1}=\delta_{+}+2 \bar{\eta}_{3} \sqrt{-\left(A B-C^{2}\right)}, & P_{2} P_{1}=-h_{3} A, \\
2 Q_{1}=\delta_{-}-2 \bar{\xi}_{3} \sqrt{-\left(A B-C^{2}\right)}, & Q_{2} Q_{1}=-h_{3} B .
\end{array}
$$

The condition $A B-C^{2}<0$ guarantees that both $P$ 's and $Q$ 's are real, so that the equality in Eq. (26) represents two intersecting straight lines in the $\xi$ - $\eta$ plane. The intersection points of these lines represent the saddles provided they are lying in the first quadrant of the plane. The classification of their heteroclinic connections can be made on the basis of those intersecting points as described below.

There are six possible distinct heteroclinic connections shown in Fig. 2 along with the corresponding solutions of Eq. (26). Among those six possibilities, Figs. 2(b) and 2(c) are obviously equivalent just as Figs. 2(d) and 2(e), since they are related by simply interchanging $\xi$ with $\eta$. It should be pointed out that except for Fig. 2(a), all those phase portraits apply for both in-gap $\left(\delta_{+} \delta_{-}<0\right)$ or out-gap $\left(\delta_{+} \delta_{-}>0\right)$ cases. In contrast, Fig. 2(a) applies for the out-gap cases only, since the fixed point at the origin is a center having $\delta_{1}>0$.

Presented in Figs. 3 is an example of the bifurcation event on the $\eta$ axis corresponding to Fig. 2(b). In this process we fixed $\bar{\xi}_{2}<0, \bar{\eta}_{2}>0$, and $\bar{\eta}_{3}>0$ while varying $\bar{\xi}_{3}$ from negative to positive value. Consequently, $S_{2 y}$ from Eq. (22) changes from negative to positive value, and $S_{3}$ from Eq. (23) changes from positive to negative value. This means that the saddle on the $y$ axis turns into a center in conjunction with the appearance of a third type fixed point as a saddle. Meanwhile, the origin remains as a center outside the gap $\left(\delta_{+} \delta_{-}>0\right)$ and there are no other fixed points than the ones mentioned.

\section{RATIONAL SOLITONS}

Let us return to the case of first class bifurcation considered earlier. For the following analysis, Eq. (8) is rewritten in the form

$$
\frac{d \phi}{d z}= \pm \sqrt{\Phi_{0}+\Phi_{1} \cos ^{2}(\phi)+\Phi_{2} \cos ^{4}(\phi)},
$$

where

$$
\begin{gathered}
\Phi_{0}=\delta_{-}^{2}+4 h B, \quad \Phi_{1}=\delta_{-}\left(\delta_{+}-\delta_{-}\right)+4 h(C-B), \\
\Phi_{2}=\left(\delta_{+}-\delta_{-}\right)^{2}+8 h(A+B-2 C) .
\end{gathered}
$$

In compliance with the second condition of Eq. (24), we must have $\bar{\eta}_{2}=0$, or $\delta_{-}=0$, hence $h_{1}=h_{2 y}=0$. Thus Eq. (29) reduces to the following simple form:

$$
\frac{d \phi}{d z}= \pm \delta_{+} \cos ^{2} \phi .
$$

For both the first and second type fixed point, the solutions are

$$
\begin{gathered}
\phi_{n}=\frac{\pi}{2}+n \pi, \\
\phi_{ \pm, n}(z)= \pm \arctan \left(\delta_{+} z\right)+n \pi,
\end{gathered}
$$

where $n=0,1$. It should be noted that the constant solutions of Eq. (31) which appear as limiting cases of Eq. (32) for 

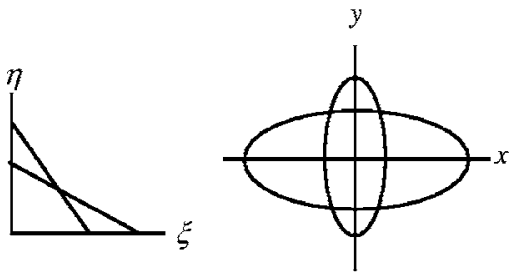

(a)
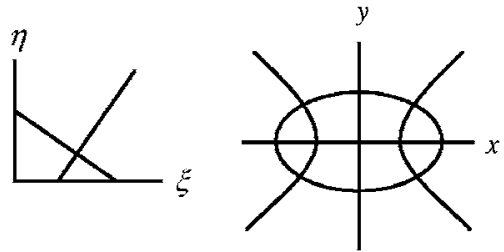

(c)

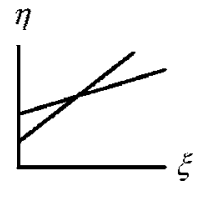

(d)
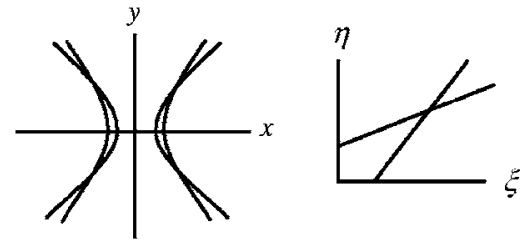

(e)
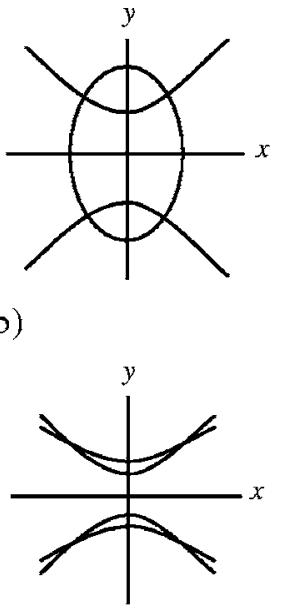

(b)

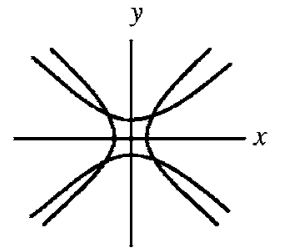

(f) $z \rightarrow \infty$ is actually associated with two isolated fixed points having $f^{2}=0$. The remaining two solutions $\phi_{-, n}(z)$ consist of the right lobe $(n=0)$ and the left lobe $(n=1)$ with the amplitude function given by

$$
f_{-}^{2}(z)=-\delta_{+}\left[\frac{1+\left(\delta_{+} z\right)^{2}}{A+2 C\left(\delta_{+} z\right)^{2}+B\left(\delta_{+} z\right)^{4}}\right] .
$$

This is a rational algebraic function, which together with the two corresponding $\phi_{-, n}(z)$ solutions represent the so called "bright-rational soliton." The associated trajectories are described in Fig. 1(b) which are characterized by the presence of a degenerate fixed point at the origin. This solitonic state appears as a result of frequency-tuning induced transition from the eight-shape trajectory in Fig. 1(a). Further increase of $\omega$ leads eventually to the heteroclinic trajectories in Fig. 1(c), featuring the appearance of two second type fixed points on the $y$ axis, with the second type fixed points remaining as centers on the $x$

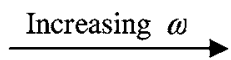

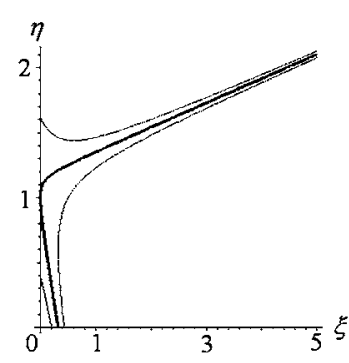

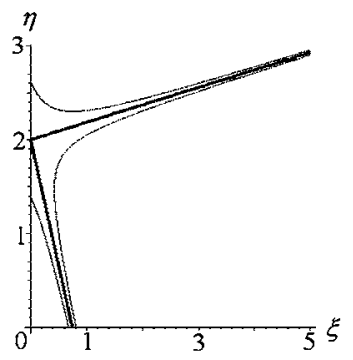

(a)
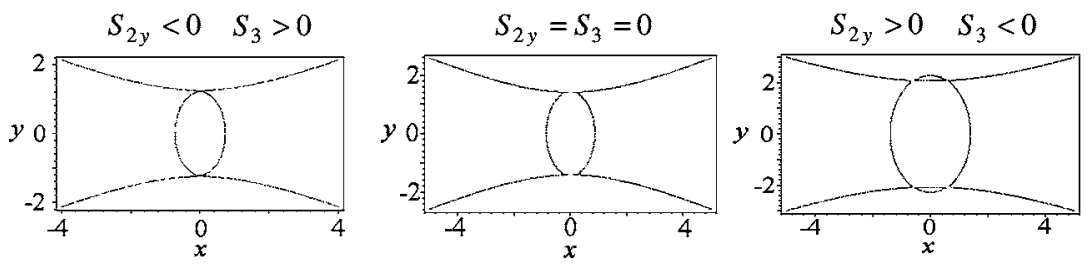

(b)

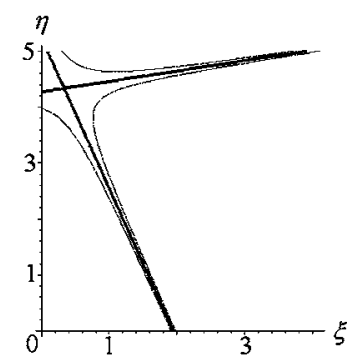

FIG. 3. A possible second class bifurcation process induced by increasing $\omega$, showing phase portraits for $h=h_{2 y}$ (left graph), $h=h_{2 y}=h_{3}$ (middle graph), and $h=h_{3}$ (right graph) in (a) $\xi-\eta$ plane (b) $x-y$ plane, corresponding to the case characterized by $A>0, B<0$, and $\delta_{+} \delta_{-}>0$, and hence $C<0$. 


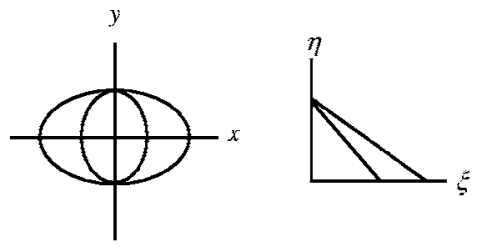

(a)

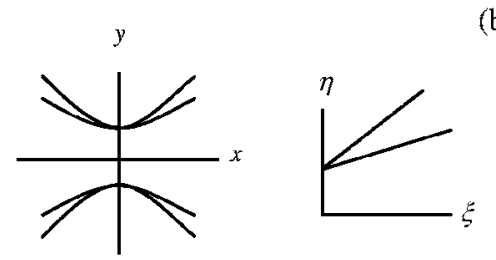

(c)

axis. Note that this interpretation hinges on the condition that $f_{-}^{2}(z)>0$ for all $z$. This is satisfied according to Eq. (33) in view of the choices made earlier, namely, $A>0, B<0$, $C>0$, and $\delta_{+}>0$.

Next, we consider the second class bifurcation events taking place on the $y$ axis only. To be specific, we consider parameter values leading to intersecting points presented in Figs. 2(a), 2(b), and 2(d) for $h=h_{3}$. As shown in Fig. 3, the second type fixed point moves from left to right, passing across the $\eta$ axis. The bifurcation happens when the second part of Eq. (25) and $\bar{\xi}_{3}=0$ are satisfied, which implies $C \delta_{-}=B \delta_{+}$according to Eq. (22). At this bifurcation point, we have $h_{3}=h_{2 y}=\delta_{-} \bar{\eta}_{2} / 2$ from Eqs. (17) and (18) as well as $\bar{\eta}_{2}=\bar{\eta}_{3}=-\delta_{-} / 2 B$ from Eqs. (14) and (15). Consequently the differential Eq. (29) simplifies to the following form:

$$
\frac{d \phi_{y}}{d z}= \pm \sqrt{D_{y}} \cos ^{2} \phi_{y},
$$

where

$$
D_{y} \equiv \delta_{+}^{2}-\delta_{-}^{2} A / B=-4 \bar{\eta}_{2}^{2}\left(A B-C^{2}\right)
$$

has a positive value since $A B-C^{2}<0$. The solutions of Eq. (34) are

$$
\begin{gathered}
\phi_{y, n}=\frac{\pi}{2}+n \pi, \\
\phi_{y, \pm, n}(z)= \pm \arctan \left(z \sqrt{D_{y}}\right)+n \pi,
\end{gathered}
$$

where $n=0,1$. The two constant solutions for $\phi_{y, n}$ yield the fixed point, $\bar{\eta}_{2}=f_{+}^{2}=f_{-}^{2}$, while the solutions $\phi_{y, \pm, n}(z)$ yields the corresponding amplitude functions:

$$
f_{y, \pm}^{2}(z)=-\frac{\delta_{-}^{2}}{2 B}\left[\frac{1+\left(z \sqrt{D_{y}}\right)^{2}}{\delta_{+} \pm \sqrt{D_{y}}+\delta_{-}\left(z \sqrt{D_{y}}\right)^{2}}\right] .
$$

The subscript $y$ indicates that the related bifurcation event occurs on the $y$ axis. Similar amplitude functions are found when the coordinates $x$ and $y$, the parameters $\delta_{+}$and $\delta_{-}$, as well as $A$ and $B$ are interchanged. Note also that $\lim _{|z| \rightarrow \infty} f_{y, \pm}^{2}(z)=-\delta_{-} / 2 B=\bar{\eta}_{2}$, implying the constant $\phi_{y, n}$ so-

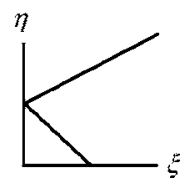

FIG. 4. The phase portraits at the bifurcation point in $x-y$ plane (left) and $\xi-\eta$ plane (right). lutions as the limiting cases for the other solutions as found earlier in the previous case.

To interpret this expression, observe that the numerator of Eq. (38) is positive for all $z$, while the denominator can have a zero at, say, $z= \pm z_{s}$, depending on the values of the parameters. In other words, $f_{y, \pm}^{2}(z)$ have singularities and change sign at $\pm z_{s}$. To characterize the behavior of the amplitude functions (38) we define the peak or dip to the pedestal ratio as $R_{ \pm}=f_{y, \pm}^{2}(z=0) / f_{y, \pm}^{2}(z= \pm \infty)$. One finds for this case,

$$
R_{ \pm}=\frac{\delta_{-}}{\delta_{+} \pm \sqrt{D_{y}}}=\frac{B}{C \mp \sqrt{-\left(A B-C^{2}\right)}} .
$$

The second equality in Eq. (39) is obtained by using the relations $\delta_{+}=-2 C \bar{\eta}_{2}$ and $\delta_{-}=-2 B \bar{\eta}_{2}$. With this, the amplitude functions (38) can be rewritten as

$$
f_{y, \pm}^{2}(z)=\bar{\eta}_{2} R_{ \pm}\left[\frac{1+\left(z \sqrt{D_{y}}\right)^{2}}{1+R_{ \pm}\left(z \sqrt{D_{y}}\right)^{2}}\right] .
$$

In the phase portrait of Fig. 4(a), the two different saddles on the $y$ axis are connected by four lines, each representing a rational dark or antidark soliton. For this case, both $R_{+}$and $R_{-}$are found to be positive so that the corresponding $f_{y,+(-)}^{2}$ are positive for $-\infty<z<\infty$, featuring the typical profiles of the solitons. The ratio, $R_{ \pm}$, for the rational dark soliton lies in the range of $0 \leqslant R_{ \pm}<1$, while $R_{ \pm}>1$ for the rational antidark soliton. For $R_{+(-)}>R_{-(+)}$, the amplitude functions $f_{y,+(-)}^{2}$ are related to the outer saddle connection, while $f_{y,-(+)}^{2}$ corresponds to the inner connection. In the phase portraits of Fig. 4(b), the two different saddles are connected by just two lines and there are four unbounded lines. In this case $R_{+}$and $R_{-}$have opposite signs. The bounded solutions are found when $R_{+(-)}>0$, while $R_{-(+)}<0$ leads to the unbounded ones associated with a zero at $z_{s}=1 / \sqrt{-R_{-(+)} D_{y}}$ in the denominator. Thus, $f_{y,+(-)}^{2}>0$ for $|z|>z_{s}$, and the relevant solutions are given by Eqs. (37) and (38) with $z$ restricted to this range. For the range $|z|<z_{s}, f_{y,+(-)}^{2}$ is negative, and $f_{y,+(-)}$ does not correspond to solution of Eqs. (1) and (2). In Fig. 4(c), all branches are unbounded, since both $R_{+}$and $R_{-}$are negative in this case. 

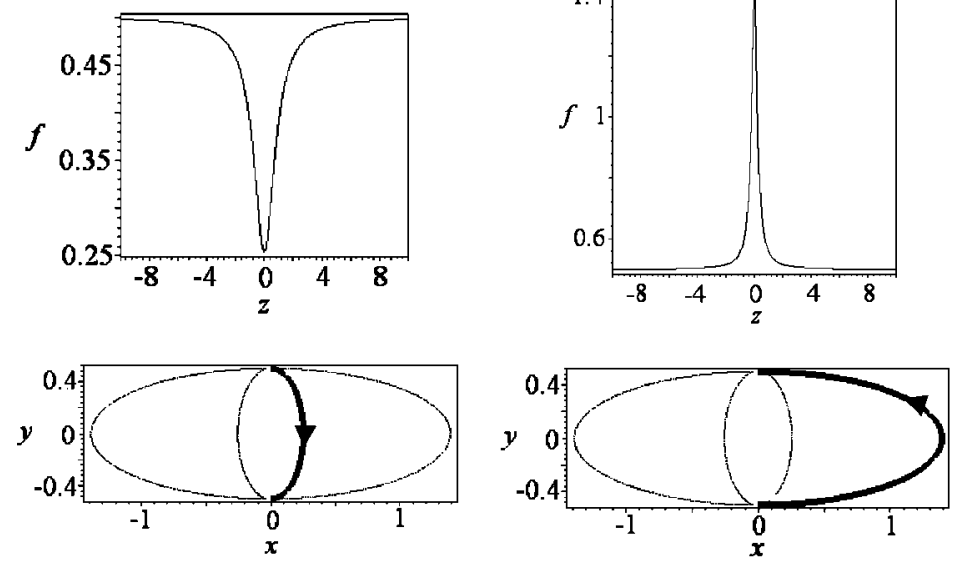

(a)
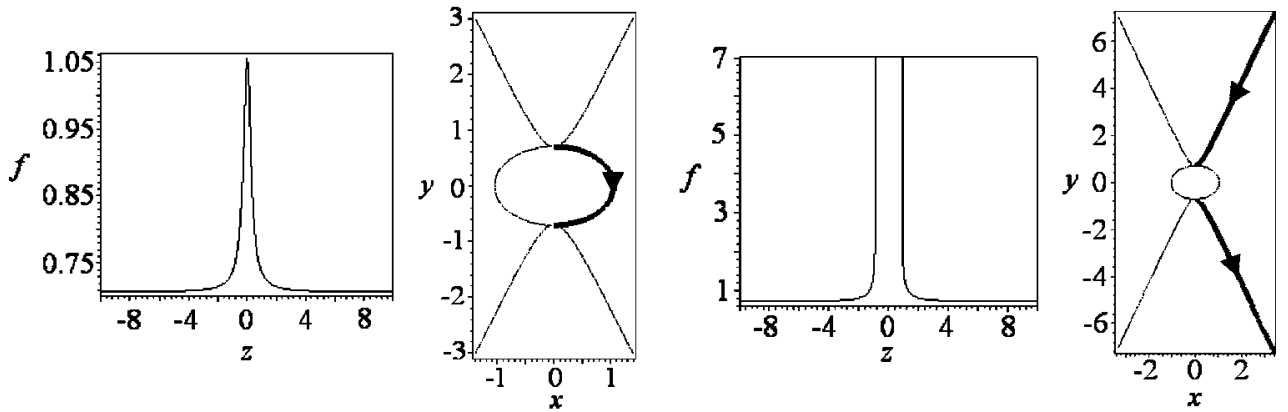

(b)

It is important to add that the phase portrait of Fig. 4(a) occurs outside the band gap only. This is the consequence of $R_{+} R_{-}=B / A>0$ and $R_{+}+R_{-}=2 C / A>0$, which implies that $A, B$, and $C$ have the same sign, and hence the condition $\bar{\xi}_{3}=0$ simply leads to $\delta_{+} \delta_{-}>0$. Similarly, with $R_{+} R_{-}=B / A>0$ and $R_{+}+R_{-}=2 C / A<0$ one readily shows that $A$ and $B$ must have the same sign while $C$ has the opposite sign, implying $\delta_{+} \delta_{-}<0$, which means that Fig. 4(c) occurs inside the band gap. For the case of Fig. 4(b), one finds $R_{+} R_{-}=B / A>0$, but $R_{+}+R_{-}=2 C / A$ can either be positive or negative. Consequently, $C$ can have the same or different sign with respect to the sign of $A$ and $B$, corresponding to $\delta_{+} \delta_{-}>0$ or $\delta_{+} \delta_{-}<0$, respectively. In other words the phase portrait of Fig. 4(b) can exist either inside or outside the band gap.

We illustrate in Fig. 5(a) the out-gap cases for a set of parameter values, showing the presence of two different saddle connections on the right half-plane. The two cases are shown to represent the dark and antidark solitons. The corresponding amplitude functions are given by $f_{y,+}^{2}$ and $f_{y,-}^{2}$, respectively for $n=0$ in Eq. (36). In Fig. 5(b) we illustrate the in-gap case for a set of parameters showing the presence of a two different saddle connections on right half-plane corresponding to the antidark soliton and the unbounded branch. The related amplitude functions are given by $f_{y,-}^{2}$ and $f_{y,+}^{2}$, respectively for $n=0$. The other orbits shown in the left half-plane of
Figs. 5(a) and 5(b) have the same amplitude functions but correspond to $n=1$.

Finally, we investigate the occurrence of rational solitons as transitional states between ordinary solitons induced by frequency detuning, which also entails corresponding variation of $f^{2}$ as implied by Eq. (7). Consider the second class bifurcation process involving solitons outside the gap. In this case $S_{2 y}$ changes from negative to positive value and $S_{3}$ from positive to negative value, as illustrated in Fig. 6(a). Prior to that process, one has a pair of ordinary out-gap dark and antidark solitons represented by trajectories connecting two saddles on the positive and negative $y$ axis and lying in the half plane on either side of the $y$ axis. As a result of increasing the frequency, these states transform via the rational soliton into two different pairs, each consisting of a dark and an antidark soliton. The first pair is associated with the two trajectories that connect the saddles in the first and fourth quadrants. Their amplitude functions feature higher background and narrower profile, compared to the amplitude functions before the bifurcation. The second pair is associated with the two trajectories connecting the saddles in the first and second quadrants. In this case, their amplitude functions grow from a flat profile related to the constant solutions prior to the bifurcation. Similar transitions related to the first class bifurcation event were discussed by Conti and Trillo [8] for moving solitons on the basis of properties of a 


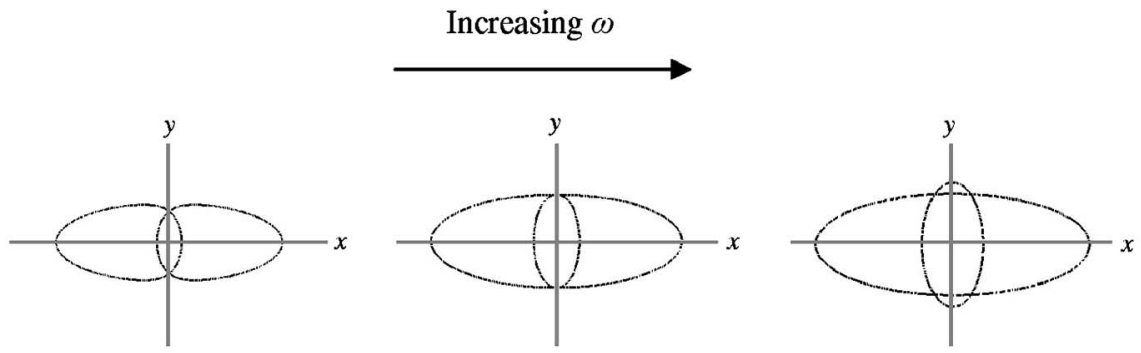

(a)

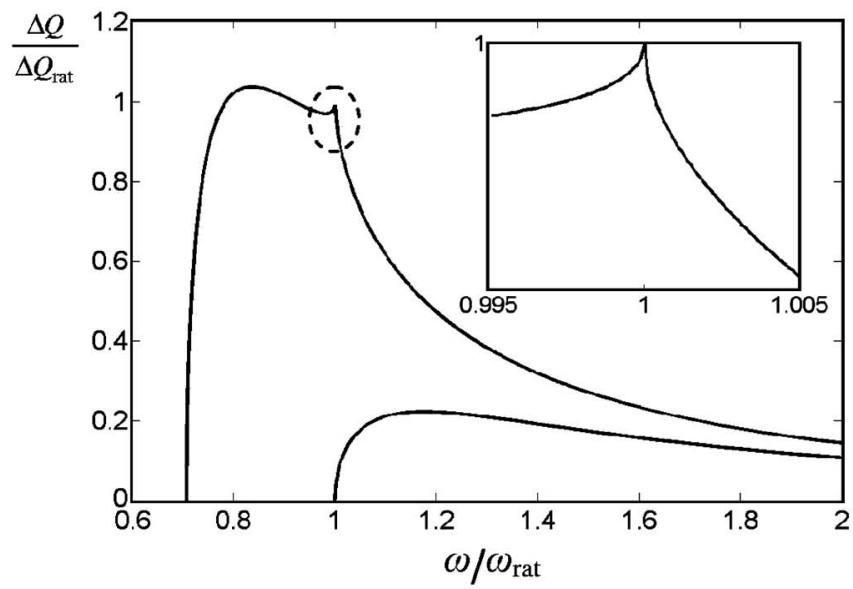

(b)

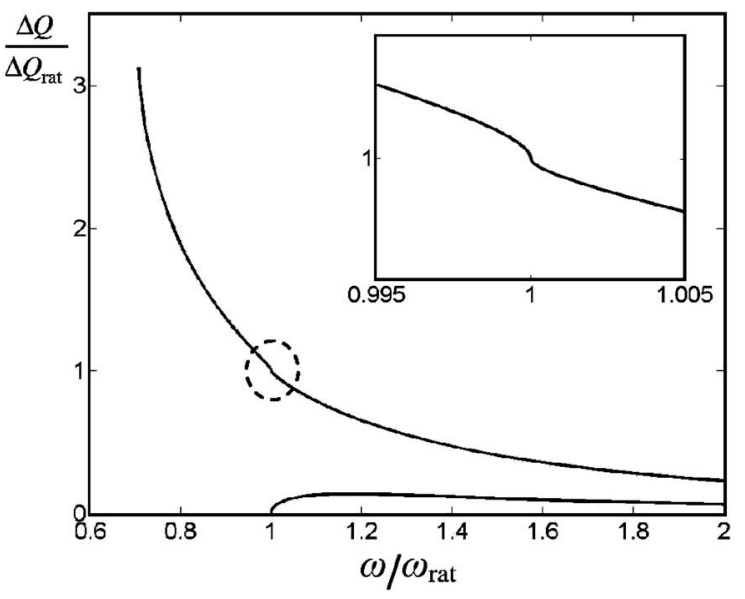

(c)

FIG. 6. (a) Evolution of the phase portraits in $x-y$ plane for increasing $\omega$, from $\omega=0.8 \omega_{\text {rat }}$ to $\omega=1.6 \omega_{\text {rat }}$, where $\omega_{\text {rat }}$ denotes the frequency at which the rational soliton occurs. The graphs in the lower section describe the frequency dependent variations of the normalized (b) loss and (c) excess intensity of the out-gap dark and antidark solitons, respectively. The changes of slope at the bifurcation point indicated on the upper branch of the curves are zoomed in the insets of each figures.

Hamiltonian like the one in Eq. (6), but with a much simpler structure.

An additional characteristic of the soliton is described by its energy profile. This profile is defined by the integral $\Delta Q(\omega)=\int_{-\infty}^{\infty} \Delta f^{2}(z, \omega) d z$, where the integrant is $\Delta f^{2}(z, \omega)=\left|f^{2}(z, \omega)-f^{2}(\infty, \omega)\right|$ and normalized to $\Delta Q_{\text {rat }}$. It is interpreted as the total energy loss (excess) of the dark (antidark) solitons with respect to the c.w. background. Numerical integrations for $\omega>\omega_{\text {rat }}$ were performed on the basis of the exact solutions of $S_{3}$ soliton found in our previous work [13]. In the present case, the values of $\Delta Q / \Delta Q_{\text {rat }}$ for $\omega<\omega_{\text {rat }}$ are calculated in the same way on the basis of exact solution of the $S_{2 y}$ soliton. The results are depicted in Figs. 6(b) and 6(c) as functions of normalized frequency $\omega / \omega_{\text {rat }}$ for different solitons encountered in the process as described above. Specifically, it is clear from each upper curve of those figures that the normalized energy exhibits a continuous variation with $\omega$ in the vicinity of $\omega_{\text {rat }}$, interrupted only at $\omega_{\text {rat }}$ by a discontinuity in its derivative. These abrupt changes further characterize the transition process induced by frequency detuning. One may also add in this connection that the soliton tails decay nonexponentially at the transition point.

It should be pointed out here that the stability analysis of these stationary rational solitons is a crucial and challenging issue that should be carefully investigated, especially for solitons with a nonvanishing background, which perhaps requires rigorous methods beyond what was given in Ref. [15]. Indeed, the oscillatory instabilities found in the in-gap bright solitons [16,17] might occur in more complicated patterns. Such stability studies are usually performed for infinite gratings. In a finite grating, which is the realistic system encountered in an experiment, the stability problem has to be formulated precisely according to the experimental setting. This creates additional difficulty in the stability analysis. It is also tempting to examine whether the Hamiltonian versus energy $(\Delta Q)$ curves, (cf. Ref. [18]), together with the properties of $\Delta Q$ described above, could yield useful results for such study. In any case, the expression found in this work may still be useful for future work.

\section{CONCLUSION}

We describe in this report the bifurcation process of solitonic solutions in a deep nonlinear Bragg grating by a geometrically simple way on the basis of the underlying Hamiltonian structure of the system. We demonstrate that the rational solitons occur at the bifurcation point as the limiting cases of ordinary solitons. Their occurrence is brought about by varying the detuning frequency, and they may appear both for frequencies inside and outside the band gap. 
The explicit expressions for these rational solitons are found in the form of rational algebraic functions of $z$. It is further demonstrated in two specific cases that in addition to in-gap bright rational soliton found previously in the case of shallow grating, both in and out-gap dark and antidark rational solitons, which were excluded in a shallow nonlinear Bragg grating, are admitted in the system considered, provided that the coefficients of the three nonlinear terms in the generalized coupled mode model satisfy a quadratic inequality. A numerical analysis of the behavior of soliton energy as a function of the frequency displays distinct features at the transition points.

\section{ACKNOWLEDGMENTS}

H.A. would like to thank the KNAW supported EPAMProject for giving him a chance to visit the University of Twente, The Netherlands. He has benefited from the stimulating environment in the Computational Material Science (CMS) Group and useful discussions with E. van Groesen of the Applied Analysis and Mathematical Physics (AAMP) Group and H. Hoekstra of the Integrated Optical Micro System (IOMS) Group of MESA+ Research Institute, where part of this work was initiated. This work is also partly supported by RUTI research project under Contract No. 2D/ PPK/RUTI/KMNRT/II/2005.
[1] W. Chen and D. L. Mills, Phys. Rev. Lett. 58, 160 (1987).

[2] B. J. Eggleton, R. E. Slusher, C. M. de Sterke, P. A. Krug, and J. E. Sipe, Phys. Rev. Lett. 76, 1627 (1996).

[3] D. Taverner, N. G. R. Broderick, D. J. Richardson, M. Ibsen, and R. I. Laming, Opt. Lett. 23, 259 (1998).

[4] D. L. Mills and S. E. Trullinger, Phys. Rev. B 36, 947 (1987).

[5] D. N. Christodoulides and R. I. Joseph, Phys. Rev. Lett. 62, 1746 (1989).

[6] A. B. Aceves and S. Wabnitz, Phys. Lett. A 141, 37 (1989).

[7] J. Feng and F. K. Kneubühl, Quantum Electron. 29, 590 (1993).

[8] C. Conti and S. Trillo, Phys. Rev. E 64, 036617 (2001).

[9] C. Conti, S. Trillo, and G. Assanto, Phys. Rev. Lett. 85, 2502 (2000).

[10] K. Hayata and M. Koshiba, Phys. Rev. E 51, 1499 (1995); J. M. Soto-Crespo, N. N. Akhmediev, and V. V. Afanasjev, Opt. Commun. 118, 587 (1995).
[11] C. M. de Sterke, D. G. Salinas, and J. E. Sipe, Phys. Rev. E 54, 1969 (1996).

[12] T. Iizuka and C. Martijn deSterke, Phys. Rev. E 61, 4491 (2000).

[13] H. Alatas, A. A. Iskandar, M. O. Tjia, and T. P. Valkering, J. Nonlinear Opt. Phys. Mater. 12, 157 (2003).

[14] A. S. Kovalev, O. V. Usatenko, and A. V. Gorbatch, Phys. Rev. E 60, 2309 (1999).

[15] C. M. de Sterke, Phys. Rev. A 45, 8252 (1992); J. Schöllmann, J. Phys. A 288, 218 (2000).

[16] I. V. Barashenkov, D. E. Pelinovsky, and E. V. Zemlyanaya, Phys. Rev. Lett. 80, 5117 (1998).

[17] A. De Rossi, C. Conti, and S. Trillo, Phys. Rev. Lett. 81, 85 (1998).

[18] A. Ankiewics and N. N. Akhmediev, IEE Proc.: Optoelectron. 150, 519 (2003). 